\title{
Synthesis and Photovoltaic Properties of Regioregular Head-to-Head Substituted Thiophene Hexadecamers
}

Francesca Di Maria, ${ }^{\dagger}$ Massimo Gazzano, ${ }^{\ddagger}$ Alberto Zanelli, ${ }^{\ddagger}$ Giuseppe Gigli, ${ }^{\S, \|, \perp}$ Anna Loiudice, ${ }^{\|}$ Aurora Rizzo, $\|, \perp$ Mariano Biasiucci, ${ }^{\#}$ Elisabetta Salatelli, ${ }^{\%}$ Pasquale D’ Angelo, ${ }^{8}$ and Giovanna Barbarella ${ }^{\ddagger} * *$

${ }^{\dagger}$ Laboratorio MIST.E-R and ${ }^{\ddagger}$ ISOF, Consiglio Nazionale Ricerche, Via P. Gobetti 101, 40129 Bologna, Italy

${ }^{\S}$ Dipartimento di Fisica e Matematica, Università del Salento, "Center for Biomolecular Nanotechnologies, Italian Institute of Technology, Energy Platform, and ${ }^{\perp}$ NNL CNR-Istituto Nanoscienze, Distretto Tecnologico, via Arnesano, 73100 Lecce, Italy

${ }^{\#}$ NNL-CNR Nanoscience Institute, c/o Dip. Fisica Ed. G. Marconi, Center for Life NanoScience, La Sapienza University and Italian Institute of Technology, Viale Regina Elena 295, 00161 Roma, Italy

${ }^{\%}$ Dipartimento Chimica Industriale e dei Materiali, Universita' di Bologna, Viale Risorgimento 4, 40163 Bologna, Italy

${ }^{8}$ IMEM, Consiglio Nazionale Ricerche, Parco Area delle Scienze 37/A, 43124 Parma, Italy

Supporting Information

ABSTRACT: We describe the expedient synthesis of regioregular thiophene hexadecamers head-to-head (hh) substituted with hexyl and hexylthio grous. The synthesis was carried out by means of a sequence of ultrasound-assisted selective monobrominations and microwave-assisted Suzuki reactions using 4,4,5,5-tetramethyl-1,3,2-dioxaborolane in THF:water. The hexadecamers, which are very soluble in organic solvents, were investigated in solution and thin film by a variety of techniques (UV, PL, CV, X-ray diffraction, FET charge mobility, SKFM) with the aim of elucidating the effect of the sulfur spacer on morphology and functional properties. We show that the sulfur spacer compensates for the decrease in $\pi-\pi$ conjugation caused by the hh regiochemistry and that the $\lambda_{\max }$ value and redox potentials of the S-alkyl-substituted hexadecamer are similar to those of head-to-tail substituted poly(3-hexylthiophene). Measurements in field effect transistor devices showed that the alkylthio-substituted hexadecamer is a p-type semiconductor while the alkyl-substituted counterpart in the same conditions is not electroactive. Scanning Kelvin force microscopy measurements showed that a blend of the alkylthiosubstituted hexadecamer with PCBM displays photovoltaic behavior under illumination. In agreement with this, a bulk heterojunction cell fabricated employing the same blend displayed near $1.5 \%$ conversion efficiency without addition of additives or device optimization.

\section{INTRODUCTION}

Considerable research effort is currently being devoted to the improvement of organic photovoltaic devices for their advantages in terms of solution processing, lightweight, flexibility, and low costs. In the past few years impressive improvements have been made, in particular in bulk heterojunction $(\mathrm{BHJ})$ cells whith power conversion efficiences now attaining over $8 \% .^{1-9,10 a, b}$ These impressive advances have been promoted by improved electron donor or acceptor functional materials, processing conditions, device engineering, and understanding of the structure-property relationship. Thiophene derivatives play a key role in the field, as either polymers or short oligomers, reaching remarkable power conversion efficiencies in $\mathrm{BHJ}$ as donors in blend with soluble fullerenes as acceptors. ${ }^{1-9,10 a, b}$

In this framework and based on our previous experience on the synthesis and application of thiophene derivatives, ${ }^{11 a, b}$ we started the search for new structural paradigms for thiophene oligomers and polymers for photovoltaic applications. The electronic properties as well as the morphology of conjugated materials are determined by their structural features, in particular their size and type and position of substituents. Therefore, syntheses leading to regioregularly substituted derivatives are required.

We focused on synthetically accessible large-size thiophene oligomers, made of more than 10 thiophene rings, as they are likely to have properties similar to those of polydisperse polymers but being monodisperse compounds are by far more soluble and easier to reprepare with exactly the same characteristics. It is worth noting that very few regioregular

Received: August 8, 2012

Revised: September 30, 2012

Published: October 11, 2012 
Scheme 1. Synthetic Pattern for the Preparation of Hexadecamers 7 and $7 a$ and the Corresponding Polymers 9 and $9 a^{a}$<smiles>[R]c1ccsc1-c1sccc1[R]</smiles>

$1,1 \mathrm{a}$<smiles>[R20]c1ccsc1-c1sc(Br)cc1[R2]</smiles><smiles>[R]c1cc(C)sc1-c1sc(C(C)(C)C)cc1[R]</smiles><smiles>[R]c1cc(C)sc1-c1sc(Br)cc1[R]</smiles><smiles>N#CC#N</smiles><smiles>[R]c1cc(C)sc1-c1sc(C)cc1[R]</smiles><smiles>CC#CCCCCCC</smiles><smiles>[R]c1cc(C)sc1-c1sc(Br)cc1[R6]</smiles><smiles>CC1CCCCC1C</smiles>

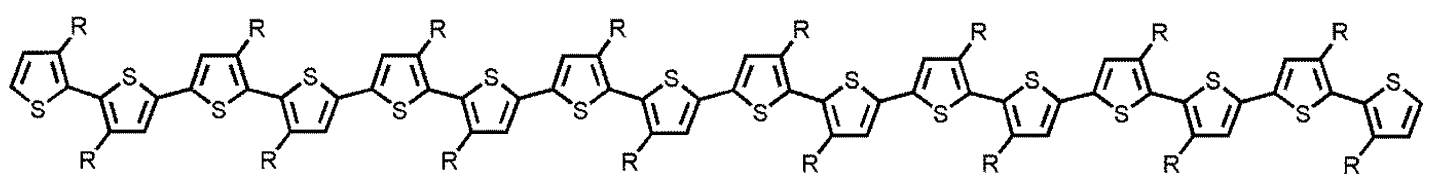

$7,7 a$

${ }^{a}$ Compounds 1-9: $\mathrm{R}=\mathrm{S}\left(\mathrm{CH}_{2}\right)_{5} \mathrm{CH}_{3}$. Compounds 1a-9a: $\mathrm{R}=\left(\mathrm{CH}_{2}\right)_{5} \mathrm{CH}_{3}$. (i) NBS 1 equiv, US, $\mathrm{RT}, \mathrm{CH}_{2} \mathrm{Cl}_{2}$; (ii) NBS 2 equiv, US, RT; (iii) 4,4,5,5-tetramethyl-1,3,2-dioxaborolane 0.6 equiv, $\mathrm{Pd}(\mathrm{dppf})_{2} \mathrm{CH}_{2} \mathrm{Cl}_{2} 5 \%, \mathrm{NaHCO}_{3} 2$ equiv, THF: $\mathrm{H}_{2} \mathrm{O} 2: 1, \mathrm{MW}, 80{ }^{\circ} \mathrm{C}$.

large-size thiophene oligomers have been described so far. ${ }^{10 c, 12 a}$ The "large size" being only limited by the possibility to prepare sizable amounts of pure oligomer, it appeared more convenient to explore thiophene compounds with substituents forming head-to-head-junctions as they are much easier to synthesize and purify than the corresponding head-to-tail oligomers. Indeed, to increase the size of head-to-tail oligomers, the oneby-one addition of substituted thiophene rings is required, ${ }^{13,14}$ while head-to-head oligomers can be doubled in size in one single step, as shown below. However, for steric reasons, headto-head junctions lead to backbone distorsions and decreased delocalization; hence, substituents must be chosen that can compensate by mesomeric effect for the loss of $\pi-\pi$ delocalization.

We report here initial results concerning a regioregular thiophene hexadecamer bearing head-to-head thioalkyl chains where the additional sulfur atoms act as a reservoir of electrons-via mesomeric effect-compensating for the decrease in electron delocalization caused by the backbone distorsions. We show that the head-to-head thioalkyl chains ensure great solubility and lead to frontier orbital energies and morphological features suitable for application of the hexadecamer in photovoltaic devices. For comparison, the synthesis and characterization of the corresponding alkylsubstituted hexadecamer are also reported.

\section{EXPERIMENTAL SECTION}

Materials. Compounds 6-9 were prepared starting from 3,3'bis(hexylthio)-2,2'-bithiophene prepared according to ref $12 \mathrm{~b}$ while compounds $6 \mathbf{a}-9 \mathrm{a}$ were prepared starting from $3,3^{\prime}$-bis(hexyl)-2,2' bithiophene prepared according to ref 11a. The detailed syntheses and characterizations are reported as Supporting Information.

Electrochemistry. All compounds were deposited by drop-casting on ITO/glass electrodes (Balzers $23 \Omega$ /square). Cyclic voltammetries (CVs) of the thin films were carried out with an AMEL 5000 electrochemical system at room temperature in a homemade three- compartment glass cell (volume $10 \mathrm{~mL}$ ) carefully purged with Ar. The supporting electrolyte was propylene carbonate $0.1 \mathrm{~mol} \mathrm{~L}^{-1}$ $\left(\mathrm{C}_{2} \mathrm{H}_{5}\right)_{4} \mathrm{NBF}_{4}$, where hexadecamers and polymers were poorly soluble. The electrochemical window of this electrolytic solution, usually assumed on Pt electrode in the range from 2 to $-2.4 \mathrm{~V}$ vs SCE, on ITO has been evaluated within 2 and $-2.15 \mathrm{~V}$. More details are provided in the Supporting Information.

X-ray Diffraction. X-ray diffraction analysis was carried out by means of a Panalytical X'Pert diffractometer equipped with a copper anode $\left(\lambda_{\text {mean }}=0.15418 \mathrm{~nm}\right)$ and a fast X'Celerator detector.

Scanning Kelvin Force Microscopy. SKFM images were acquired with a commercial AFM system (Bruker-AXS) MultiMode AFM with a Nanoscope V controller operating in lift mode (typical lift height $20 \mathrm{~nm}$ ) by using silicon tips with PtIr coating (SCM- PIT) with $k \approx 3 \mathrm{~N} \mathrm{~m}^{-1}$, tip radius $\approx 20 \mathrm{~nm}$, and resonant frequency $\approx 80 \mathrm{kHz}$.

Field-Effect Transistors. Solutions of $3 \mathrm{mg} / \mathrm{mL}$ in chlorobenzene were prepared in a controlled environment (glovebox) and stirred all night at $600 \mathrm{rpm}$ on a hot plate $\left(60{ }^{\circ} \mathrm{C}\right)$. Active channels of OFET devices, in both bottom contact-bottom gate (BC-BG) and bottom contact-top gate (BC-TG) architectures, were prepared by spincoating the as-prepared solutions. Before $\mathrm{Al}$ gate electrode deposition (20 nm thick), thermally evaporated on CYTOP, the devices were annealed at $100{ }^{\circ} \mathrm{C}$ for $2 \mathrm{~h}$. More details in are given in the Supporting Information.

Bulk Heterojunction Solar Cells. Hexadecamers 7 and 7 a were tested in photovoltaic devices with structure ITO/poly(3,4ethylenedioxythiophene):poly(styrenesulfonate) (PEDOT:PSS) (40 $\mathrm{nm})$ /oligomer:PCBM $(\sim 100 \mathrm{~nm}) / \mathrm{Al}(150 \mathrm{~nm})$. ITO glass substrates were sequentially cleaned by ultrasonication in water, acetone, and isopropyl alcohol then were cleaned for $10 \mathrm{~min}$ at $85{ }^{\circ} \mathrm{C}$ using TL-1 solution cleaner. The ITO surface was then modified by spin-coating of a conductive poly(3,4-ethylenedioxythiophene)-poly(styrenesulfonate) (PEDOT-PSS) thin film $(40 \mathrm{~nm})$ followed by a baking at $140{ }^{\circ} \mathrm{C}$ in a nitrogen atmosphere for $15 \mathrm{~min}$. The active layer composed by a blend solution of oligomer: $[6,6]$-phenyl-C61-butyric acid methyl esther (PCBM, Nano-C,1:0.8 wt \% in chlorobenzene) was spin-coated at $500 \mathrm{rpm}$ for $15 \mathrm{~s}$ and dried under a Petri dish. All the operations concerning the deposition of the active layer were performed in a glovebox under a nitrogen atmosphere. The $\mathrm{Al}$ 
electrodes (ca. $100 \mathrm{~nm}$ ) were thermally evaporated at low pressure $(<7$ $\times 10^{-6}$ Torr) through a shadow mask obtaining $0.03 \mathrm{~cm}^{2}$ device areas. The device testing was carried out in ambient conditions under illumination of 1 sun (AM 1.5G). Atomic force microscopy images were acquired with a XE-100 PSIA Park scanning probe microscope in noncontact mode.

\section{RESULTS AND DISCUSSION}

The molecular structures of the head-to-head hexadecamers described in this study are shown in Scheme 1. For the correct definition of substitution patterns see ref $11 \mathrm{c}$.

In studying new synthetic materials for photovoltaic applications, numerous characterizations are required to establish a sound structure-property relationship: from optical features and charge transport properties to the position of frontier orbitals and aggregation modalities in thin film as well as application in prototype devices.

To avoid the fragmentation of the results in different papers and give a thorough description of the new synthetic materials, many details of the different characterizations have been reported as Supporting Information.

Synthesis and Optical Properties. Scheme 1 illustrates the synthesis of the hexadecamers starting from 3,3'-bis(hexylthio)-2,2'-bithiophene and 3,3'-bis(hexyl)-2,2'-bithiophene. Dimer 1 was prepared according to the methodology described in ref $12 \mathrm{~b}$ in high yield (70\%). Application of the same methodology failed to afford quaterthiophene 3 in reasonable yield.

The preparation of the hexadecamers consists of a succession of ultrasound assisted monobromination reactions and one-pot borylation-Suzuki coupling reactions using commercial 4,4,5,5tetramethyl-1,3,2-dioxaborolane in THF:water under microwave irradiation. The yields in pure monobromo derivative (>99\% from ${ }^{1} \mathrm{H}$ NMR) of the octamers were $35 \%$ for 6 and $60 \%$ for 6 a upon sonication for $15 \mathrm{~min}$ in methylene chloride and separation by silica gel chromatography. The reaction of the monobromo octamers with the dioxaborolane to obtain hexadecamers 7 and 7a occurs in reasonably good yields, $60 \%$ and $80 \%$, respectively. More details are given in the Supporting Information. To test the synthetic methodology in the preparation of the polymers of dimers 1 and 1a, the dibromo derivatives were reacted with 4,4,5,5-tetramethyl-1,3,2-dioxaborolane in THF:water under microwave assistance. The corresponding head-to-head polymers 9 and 9a were obtained in $20 \%$ and $30 \%$ yields in $60 \mathrm{~min}$. Attempts to optimize this reaction are currently under way.

Oligomers and polymers were analyzed by GPC using polystyrene standards to estimate $M_{\mathrm{n}}$ (number-average molecular weight) and $M_{\mathrm{w}}$ (mass-average molecular weight). The ratio $M_{\mathrm{w}} / M_{\mathrm{n}}$ gives a measure of polydispersity, which is 1 in the case of oligomers for which $M_{\mathrm{n}}$ and $M_{\mathrm{w}}$ are the same. Figure $1 \mathrm{~S}$ shows the GPC plots of head-to-head dimers, tetramers, octamers, and hexadecamers of 3-(hexylthio)thiophene and 3-(hexyl)thiophene, differing from each other for the retention time which is shorter for the longer oligomers. The GPC plots of polymers 9 and $9 a$ indicate polydispersity values of 2.06 and 1.7, respectively, and average degree of polymerization values (with respect to a single thiophene unit) of 55 and 52, respectively. The details are reported in the Supporting Information.

Figure 2 shows the absorption (A) and the photoluminescence (B) spectra of compounds 1-7 and 1a-7a. The absorption and photoluminescence spectra of polymers 9 and $9 \mathrm{a}$ are shown in Figure 8S. The absorption and photoluminesce wavelengths of all compounds are given in Table 1S. Figure 1 and Table $1 S$ show that the $\lambda_{\max }$ values of

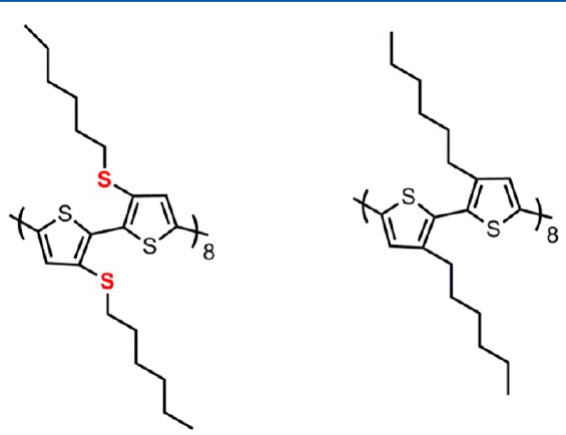

Figure 1. Molecular structure of the thiophene hexadecamers described in this study.

compounds 1-7 are remarkably red-shifted with respect to those of compounds $1 \mathbf{a}-7 \mathbf{a}$, indicating that the extra sulfurs contribute in a significant way to the electron delocalization of the aromatic skeleton.

On increasing the oligomer size, the maximum wavelength absorption increases and the molar absorption coefficients $(\varepsilon$, $\mathrm{cm}^{-1} \mathrm{~mol}^{-1}$ ) double (or more): 95.069 (7), 47.713 (5), 14.839 (3), 7.397 (1); 98.724 (7a), 51.161 (5a), 24.195 (3a), 11.957 (1a). To our knowledge, the $\varepsilon$ values for hexadecamers 7 and $7 \mathrm{a}$ are the highest ever measured for thiophene oligomers.

All compounds are characterized by large Stokes shifts from absorption to emission, as shown in Table 1S. On increasing the size of the hexylthio-substituted oligomers, $\lambda_{\mathrm{PL}}$ changes from 417 to $586 \mathrm{~nm}$, while the corresponding values for the hexyl-substituted oligomers change from 378 to $541 \mathrm{~nm}$. Moreover, for both polymers $\lambda_{\max }$ and $\lambda_{\mathrm{PL}}$ are similar to those of the corresponding hexadecamers, an indication that in the polymers the maximum conjugation length is close to that of the hexadecamers.

Cyclic Voltammetry. Figure $3 \mathrm{~A}$ shows the second voltammograms at $0.1 \mathrm{~V} \mathrm{~s}^{-1}$ of solid films in propylene carbonate $0.1 \mathrm{~mol} \mathrm{~L}^{-1}\left(\mathrm{C}_{2} \mathrm{H}_{5}\right)_{4} \mathrm{NBF}_{4}$ of hexadecamers $7,7 \mathbf{a}$ and, for comparison, of polymers $\mathbf{9}, \mathbf{9 a}$.

Oxidation waves are reversible whereas reduction waves are clearly irreversible, only polymer 9 showing a reverse peak in the cathodic region (about $-1.6 \mathrm{~V}$ ). This polymer shows fair reversibility in the anodic region and an oxidation maximum at $1.15 \mathrm{~V}$, higher than that already reported for electrochemically synthesized poly(3,3'-dialkylsulfanyl-2,2'-bithiophenes). ${ }^{15}$ Figure $3 \mathrm{~B}$ shows the onset potentials, ionization potentials (IP), and electron affinities (EA) of hexadecamers 7, 7a and polymers 9, 9a. The figure also shows the maximum wavelength absorptions $\left(\lambda_{\max }\right)$, energy gaps evaluated from both electrochemical $\left(E_{\mathrm{g}} \mathrm{ec}^{\mathrm{c}}\right)$ and spectroscopic data, ${ }^{16,17}$ and the maximum wavelenghts of the photoluminescence spectra. IP and EA values of the solid films reported in Figure $3 \mathrm{~B}$ have been estimated from the onset of the oxidation and reduction waves, respectively. ${ }^{17}$

It is seen that on going from hexadecamer $7 \mathbf{a}$ to hexadecamer 7 the presence of the extra sulfurs causes a $0.38 \mathrm{~V}$ decrease of $E_{\text {ox }}^{\text {onset }}$ while the $E_{\text {red }}^{\text {onset }}$ shifts to a $0.33 \mathrm{~V}$ less negative value with an overall energy gap decrease of more than $0.6 \mathrm{~V}$. Figure 3 also shows than on going from hexadecamer $7 \mathrm{a}$ to hexadecamer 7 , there is a very large increase in the maximum absorption 


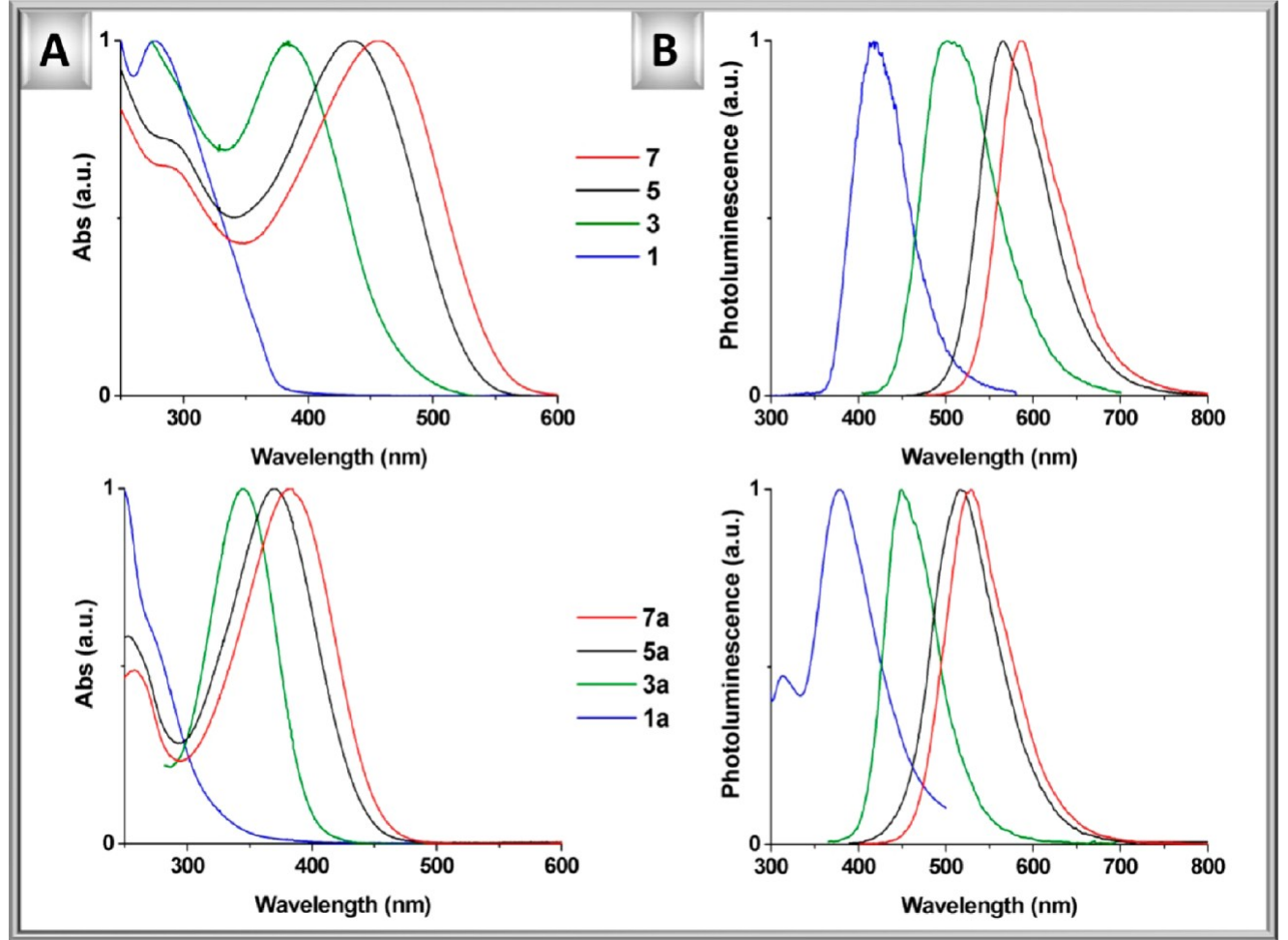

Figure 2. (A) Absorption and (B) photoluminescence spectra in $\mathrm{CH}_{2} \mathrm{Cl}_{2}$ of dimers 1, 1a, tetramers 3, 3a, octamers 5, 5a, and hexadecamers 7, 7a.

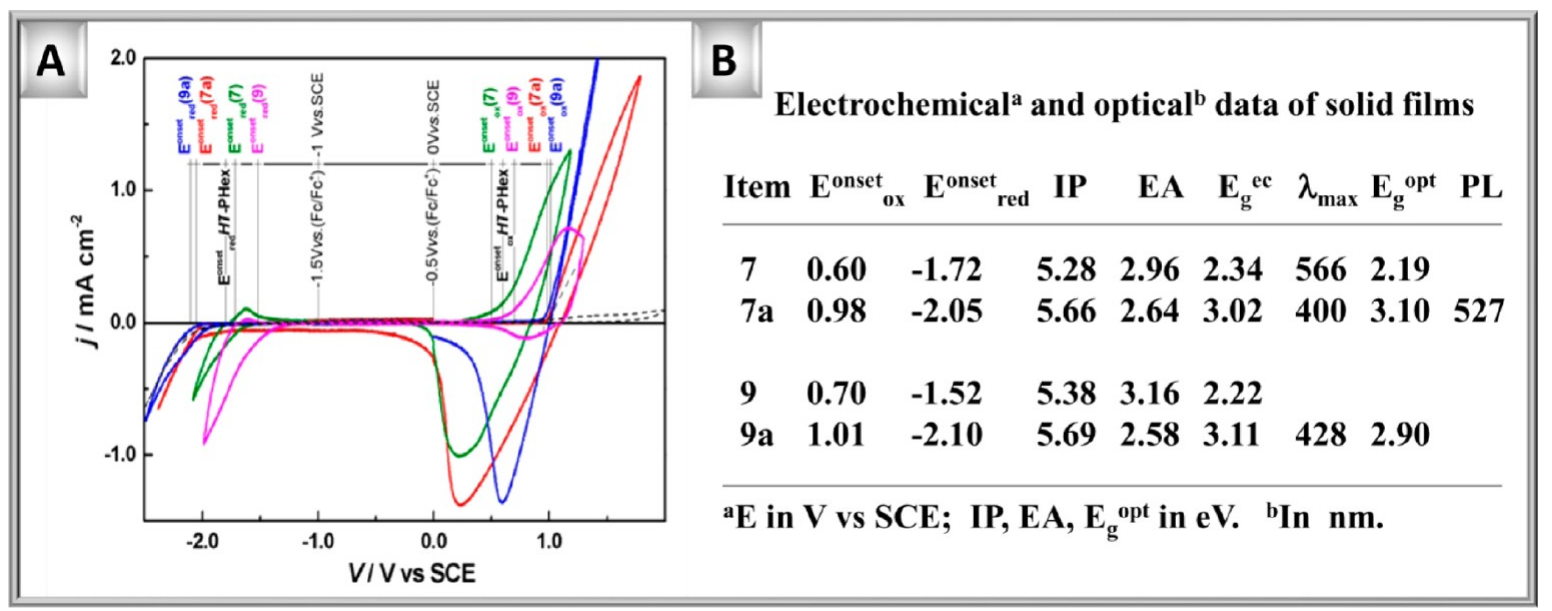

Figure 3. (A) Second cyclic voltammograms in propylene carbonate $0.1 \mathrm{~mol} \mathrm{~L}^{-1}\left(\mathrm{C}_{2} \mathrm{H}_{5}\right)_{4} \mathrm{NBF}_{4}$ at $0.1 \mathrm{~V} \mathrm{~s}^{-1}$ of hexadecamers 7 , 7a and polymers 9 , 9a (green, orange, pink, and blue traces, respectively). The horizontal scale compares their onset potentials to that of poly(3-hexylthiophene) after Hamaguchi ${ }^{18}$ both vs SCE and vs $\mathrm{Fc} / \mathrm{Fc}^{+}$. (B) Electrochemical and optical data of solid films.

wavelength of the corresponding thin films, from 400 to 566 $\mathrm{nm}$, once again ascribable to the electronic effect of the extrasulfurs. On going from the hexadecamers to the corresponding polymers $E_{\mathrm{ox}}^{\text {onset }}$ increases by $0.1 \mathrm{~V}$ from 7 to 9 and $0.03 \mathrm{~V}$ from $7 \mathbf{a}$ to $9 \mathrm{a}$ while $E_{\text {red }}^{\text {onset }}$ become less negative by 0.2 and $0.05 \mathrm{~V}$, respectively. Both $E_{\mathrm{ox}}^{\text {onset }}$ and $E_{\text {red }}^{\text {onset }}$ for the sulfur overrich hexadecamer are close to the corresponding values reported in acetonitrile $0.1 \mathrm{~mol} \mathrm{~L}^{-1}\left(\mathrm{C}_{4} \mathrm{H}_{9}\right)_{4} \mathrm{NBF}_{4}$ for head-totail poly(3-hexylthiopene) obtained by radical polymerization with $\mathrm{FeCl}_{3} \cdot{ }^{18}$ It is also worth noting that hexadecamer 7 is 46 $\mathrm{nm}$ red-shifted with respect to head-to-tail poly(3-hexylthiophene) electrochemically synthesized. ${ }^{19}$

X-rays and Morphology. The X-ray diffraction profiles of cast films from toluene of hexadecamers $7 \mathbf{a}$ and 7 are reported in Figure 4 (traces a and b, respectively). The films were highly stable and did not show any change after months from preparation. It is seen that $7 \mathbf{a}$ is much more crystalline than its sulfur overrich counterpart 7 , which displays a mix of crystalline and amorphous morphology. In the hope to improve the crystallinity of the film of hexadecamer 7 , we prepared a film cast from toluene under acetonitrile vapors, employing the methodology affording highly crystalline helical fibers of the corresponding octamer. ${ }^{11 a}$ However, the film obtained in this way showed essentially the same profile as the film simply cast from toluene. The same mixed morphology is also shown by the film of the blend of 7 with fullerene (prepared for the fabrication of the BHJ solar cell, see below), as shown by Figure $4 \mathrm{c}, \mathrm{d}$. 


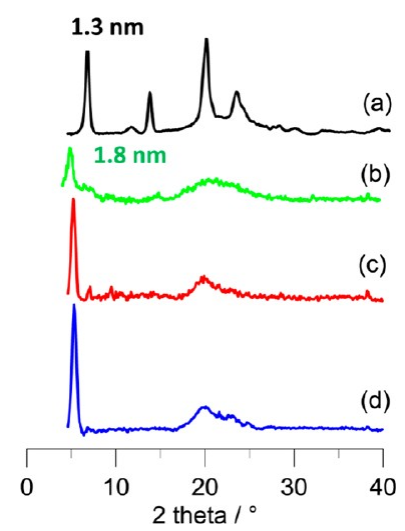

Figure 4. X-ray diffraction profile of films of hexadecamer 7a (a) and 7 (b) and of the blend of 7 with PCBM obtained in air (c) or under acetonitrile vapors $(\mathrm{d})$.

For the sulfur overrich octamer we assumed a planar conformation in the solid state, ${ }^{11 a}$ driven by intra- and intermolecular S...S interactions. In the ordered aggregates of hexadecamer 7 the situation is likely to be more complicated owing to the much larger size of the oligomer and the balance between the disorder induced by the alkyl chains and the tendency to planarity driven by sulfur-sulfur interactions. It is to note that the marked decrease in energy gap and the large increase in maximum absorption wavelength observed in the thin films of 7 compared to $7 \mathbf{a}$ (lacking the extrasulfurs) are in agreement with the hypothesis of a planar conformation for the former hexadecamer.

A temptative model for the solid state organization of $7 \mathbf{a}$ and 7 accounting for $\mathrm{X}$-ray diffraction interlayer distances is proposed in Figure 9S.

Charge Transport. To test the semiconducting properties of the hexadecamers, field effect transistor (FET) devices were fabricated (see Supporting Information).

Compound 7 showed a p-type semiconducting behavior with FET charge mobility up to $9.5 \times 10^{-4} \mathrm{~cm}^{2} \mathrm{~V}^{-1} \mathrm{~s}^{-1}$, whereas in the same conditions the FET charge mobility of $7 \mathbf{a}$ (as well as that of polymers 9 and $9 a$ ) was under the limits of detectability. Whether this result is to ascribe to electronic or morphological reasons or to a different orientation of the molecules on the substrate or, more probably, to the interplay of all different factors is difficult to establish. More detailed investigations on this precise point would be required, which, however, are beyond the aim of the present study.

Table 1 shows the relevant parameters of the field-effect transistors fabricated with 7 as the active material vacuum deposited on $\mathrm{SiO}_{2}$ or CYTOP (an amorphous fluoropolymer widely used as organic gate dielectric in organic field-effect transistors due to its excellent surface properties ${ }^{20}$ ), as gate

Table 1. Charge Mobility, Threshold Voltage, Onset Voltage, and On-Off Ratio Calculated for BC-BG Field-Effect Transistors Having $\mathrm{SiO}_{2}$ and CYTOP as the Gate Dielectric and Hexadecamer 7 as the Active Material

$\begin{array}{llccc} & \text { mobility }\left(\mathrm{cm}^{2} \mathrm{~V}^{-1} \mathrm{~s}^{-1}\right) & V_{\text {th }}(\mathrm{V}) & V_{\text {on }}(\mathrm{V}) & I_{\text {on }} / I_{\text {off }} \\ \mathrm{SiO}_{2} & 2.8 \times 10^{-5} \text { linear } & +5.5 & +10 & \sim 10^{3} \\ & 3.4 \times 10^{-5} \text { saturation } & +9 & +11 & \sim 10^{4} \\ \mathrm{CYTOP} & 5.1 \times 10^{-4} \text { linear } & -27 & -12 & \sim 10^{4} \\ & 9.5 \times 10^{-4} \text { saturation } & -26.6 & -13 & \sim 10^{5}\end{array}$

dielectrics. Output plots and transfer characteristics are reported in Supporting Information (Figure 10S). Table 1 shows that when the less hydrophilic CYTOP is employed the charge carrier mobility is improved by 1 order of magnitude in linear regime and almost 2 orders of magnitude in saturation mode with respect to the values obtained using $\mathrm{SiO}_{2}$ as gate dielectric. The enhanced mobility, the lack of hysteresis, and the threshold voltage shift toward negative gate values indicate that the quality of the gate dielectric/organic semiconductor interface, rather than the material purity, plays a significant role in terms of device operation.

It is worth noting that the FET charge mobility values measured for hexadecamer 7 are in the range of those measured for P3HT and are of the same order of magnitude as those recently obtained for a a donor-acceptor thiophene-based polymer reaching $7.3 \%$ efficiency in a $\mathrm{BHJ}$ cell. ${ }^{21}$

Scanning Kelvin Force Microscopy (SKFM). A film 30 $\mathrm{nm}$ thick of a blend of hexadecamer 7 with PCBM (1:0.8 w:w, spinned from chlorobenzene on ITO) was analyzed by SKFM in order to correlate morphological and surface electronic properties and obtain photoinduced surface photovoltage (SP) maps of the film.

Figure 5 shows the surface potential images calculated from the equation $\mathrm{SP}=\phi_{\text {sample }}-\phi_{\text {tip }}{ }^{22}$ measured by calibrating the cantilever on a graphite (HOPG) reference sample. ${ }^{23}$ A work function of $5.29 \pm 0.02$ was measured for $\phi_{\text {tip }}$ which is consistent with values reported in other works. ${ }^{24}$

Figure 5a,b shows atomic force microscopy images (topography, phase) and Figure 5d-f SKFM surface potential images of the film. The topography (a) and phase (b) images show that the blend consists of 100-250 nm sized clusters in a matrix of hexadecamer 7 . In the phase image two different kinds of clusters can be identified: those with bright orange color and those with blue color in the phase scale of values. The phase difference between the clusters is due to the presence (blue) or absence (bright orange) of a layer of 7 covering the PCBM clusters. This difference is also evident in the SP measurements. Figure 5d shows uncovered PCBM clusters with lower value of SP and covered clusters with medium (green) value of SP; higher values (blue) of SP correspond to the 7-rich matrix. SP images over the same area of the sample in three different condition of illuminations (Figure 5d-f) were acquired. By comparing the SP images with topographical and phase images, we are able to identify the regions with lower surface potential as PCBM rich aggregates, the lighter regions as 7-rich matrix, and the green regions as belonging to the interface of PCBM with hexadecamer 7.

Since in the dark charges are thought to be absent in the organic semiconductor, the surface potential should be equal to the work function difference between the tip and the ITO electrode. In our experiment the ITO work function is $\phi_{\text {Ito }} \approx$ $4.7 \mathrm{eV}^{25}$ and the estimated difference $\phi_{\text {Ito }}-\phi_{\text {Tip }} \approx-0.59 \mathrm{eV}$. In the dark (Figure 5d) the 7-PCBM region shows a contrast in the surface potential map (Figure $5 \mathrm{e}$ ) and potential distribution (Figure 5e) probably due to band bending at the interface with the substrate and consequently diffusion from bottom ITO electrode of holes in the 7 rich and electrons in the PCBM rich regions. Under the illumination photovoltaic behavior is shown by the blend. Exciton dissociations occur at the interface with an increase of the surface potential versus higher surface potential values. The positive shift of the surface potential corresponds to an excess of holes on the surface. As soon as the light is switched off, all photogenrated charges should escape 

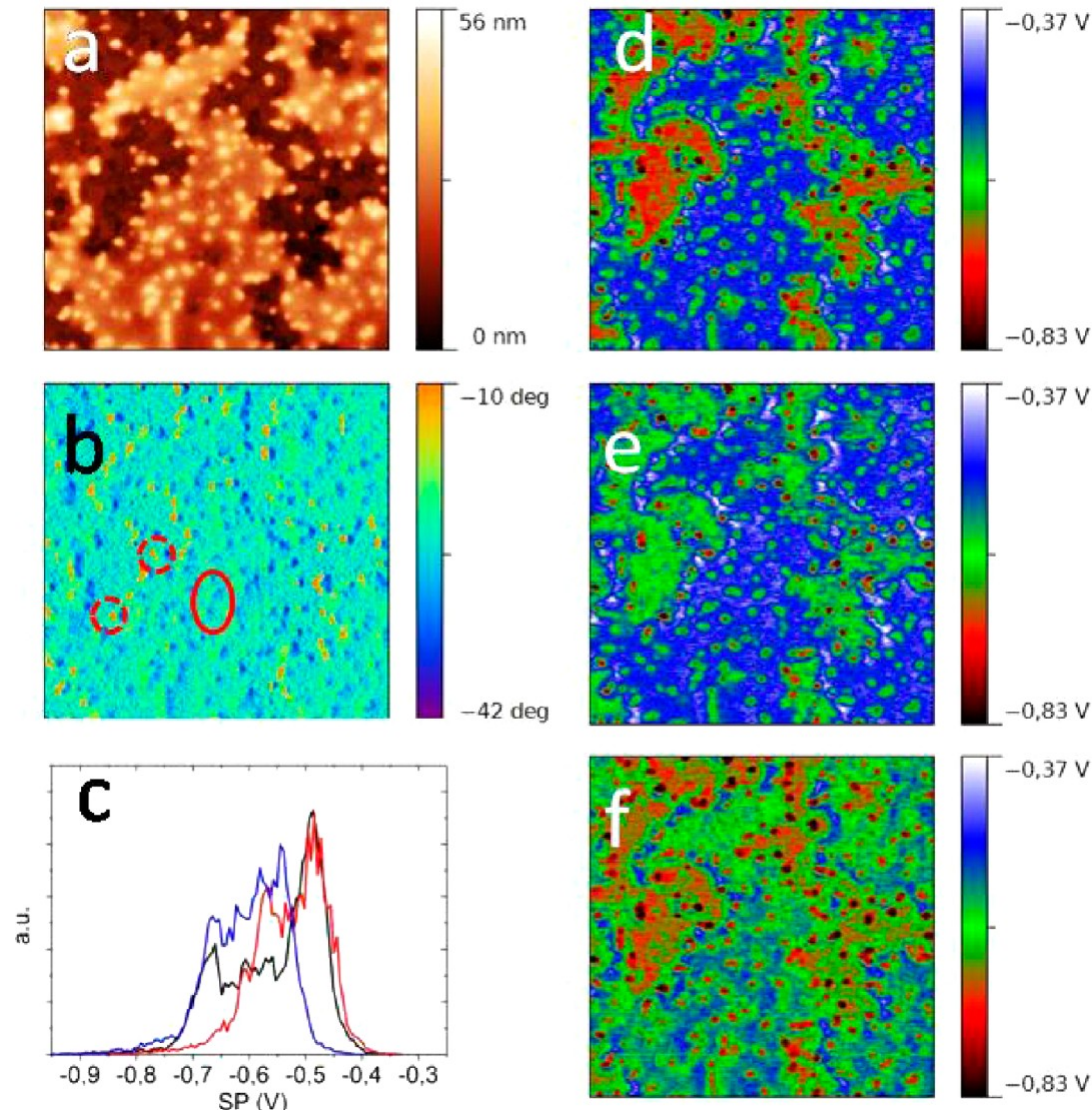

Figure 5. Atomic force microscopy (a) and phase images (b) of 7-PCBM blend. Topography and phase images show PCBM aggregates mixed in a 7 matrix. Light blue-green and bright orange colors indicate PCBM aggregates uncovered (dashed circle) and covered (continuos circle) by 7 (b). (c) Histogram representation of the surface potential distribution in the dark $(d, f)$ and under illumination (e) for the 7-PCBM blend. The histograms show a representative example of the potential distribution measured on a single $5 \times 5 \mu \mathrm{m}$ area. Thick black, red, and blue lines indicate dark 1 (before illumination), light, and dark 2 (after illumination). Surface potential images of the same area of 7-PCBM blend on ITO before (d), under (e), and after (f) illumination.

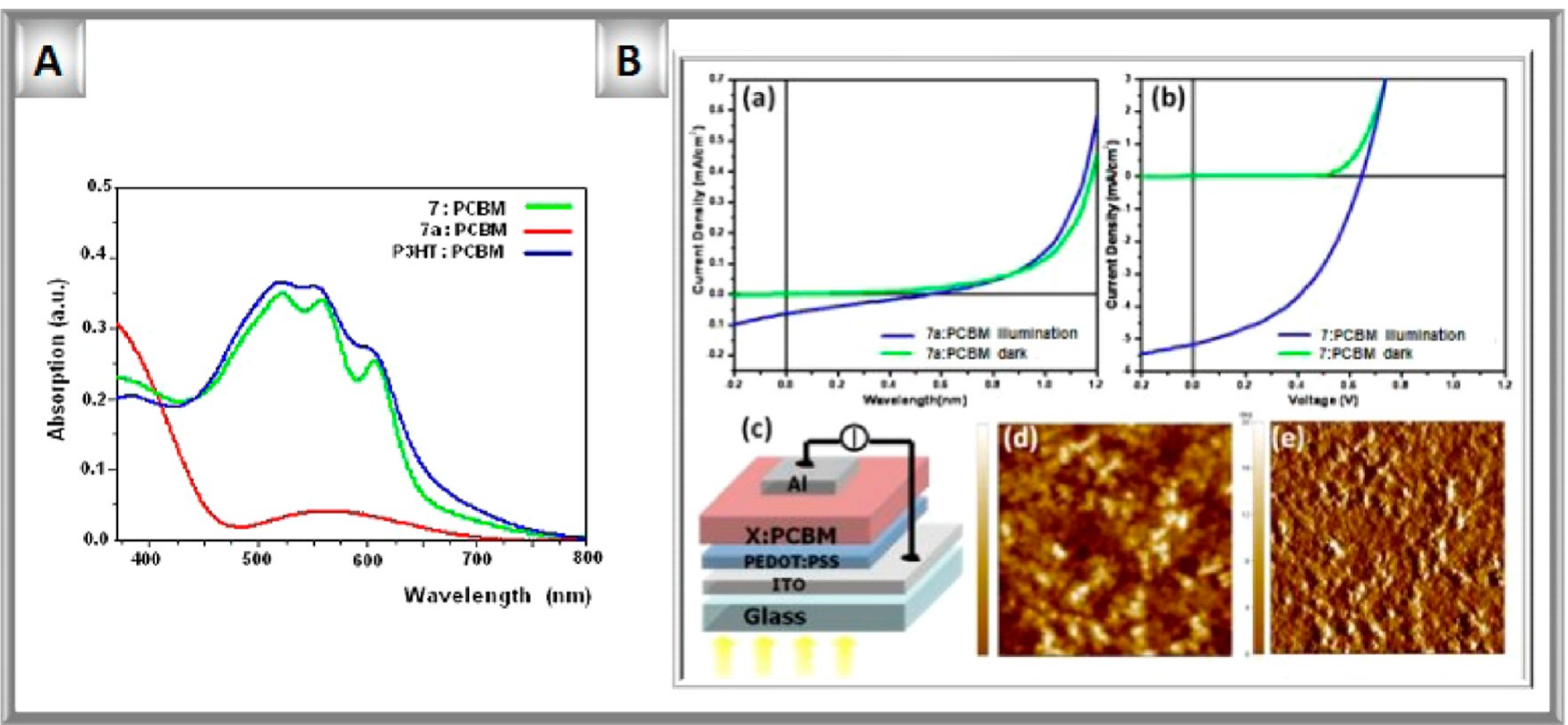

Figure 6. (A) Absorption spectra of devices with 7 (green), 7a (red), and P3HT (blue) for a film thickness of about $80 \mathrm{~nm}$. (B) Current densityvoltage characteristics under illumination (blue line) and in the dark (green line) of the device with the hexadecamer 7a (a) and 7 (b). (c) Schematic representation of the device structure, (d) AFM topography of the active layer with the hexadecamer 7, and (e) the relative phase image, scan size 5 $\times 5 \mu \mathrm{m}$. 
Table 2. Summary of Properties of the BHJ Solar Cell Prepared with Hexadecamers 7 and 7 a

\begin{tabular}{ccccccc} 
& $\eta(\%)$ & FF & $V_{\mathrm{OC}}(\mathrm{V})$ & $J_{\mathrm{SC}}\left(\mathrm{mA} / \mathrm{cm}^{2}\right)$ & $R_{\mathrm{p}}\left(\Omega \mathrm{cm}^{2}\right)$ & $R_{\mathrm{s}}\left(\Omega \mathrm{cm}^{2}\right)$ \\
7:PCBM & 1.49 & 0.46 & 0.64 & 5.13 & $1.2 \times 10^{5}$ & 59 \\
7a:PCBM & 0.01 & 0.25 & 0.56 & 0.06 & $0.9 \times 10^{5}$ & $1.1 \times 10^{4}$ \\
\hline
\end{tabular}

via the bottom electrode. A quasi-reversible potential shift between dark 1 and dark 2 is shown (Figure 5c).

Bulk Heterojunction Solar Cells. Hexadecamers 7 and $7 \mathrm{a}$ were tested in $\mathrm{BHJ}$ devices as active media blended with PCBM in a 1:0.8 ratio by weight. Figure $6 \mathrm{~A}$ compares the UV-vis spectra of the blends 7-PCBM and 7a-PCBM with the blend P3HT-PCBM. Remarkably, the absorption spectrum of the blend 7-PCBM is superimposable to the spectrum of the blend P3HT-PCBM and shows better resolved vibronic side bands, in particular the more pronounced shoulder centered at $607 \mathrm{~nm}$, which has been assigned to a highly interchain-delocalized excitation. ${ }^{26}$ This suggests that, as P3HT and in agreement with $\mathrm{X}$-ray diffraction data, 7 self-assembles during the deposition to form crystallites. Since the IP and EA values for hexadecamer 7 are very similar to those of $\mathrm{P} 3 \mathrm{HT}$, which guarantees an efficient charge separation at the interface with PCBM, one can expect a reasonably good performance for the corresponding solar cell. On the contrary, this will not be the case of hexadecamer $7 \mathbf{a}$, whose blend with PCBM displays a peak in the visible much less intense than that of 7 (which is likely to cause a substantial reduction in the $J_{S C}$ and in the overall performances of the device) and has IP and EA values very different from those of P3HT.

A sketch of the solar cell structure and the performances for the two materials are reported in Figure 5 and summarized in Table 2.

The device based on 7 a showed an open circuit voltage $V_{\mathrm{OC}}$ of $0.56 \mathrm{~V}$, a short circuit current density $J_{\mathrm{SC}}$ of $0.6 \mathrm{~mA} \mathrm{~cm}{ }^{-2}$, and a fill factor FF of 0.25 , leading to a PCE of only $0.01 \%$ under simulated solar illumination in AM1.5G conditions. On the contrary, the device based on 7 delivered much superior performances with a $V_{\mathrm{OC}}$ of $0.64 \mathrm{~V}$, a $J_{\mathrm{SC}}$ of $5.13 \mathrm{~mA} \mathrm{~cm}^{-2}$, and a FF of 0.46 , thus improving the PCE up to $1.49 \%$. The series resistance $\left(R_{\mathrm{s}}\right)$ and shunt resistance $\left(R_{\mathrm{sh}}\right)$, calculated from the inverse slope of $J-V$ characteristics at $V=0 \mathrm{~V}$ and $V=\mathrm{V}_{\mathrm{OC}}$, respectively, are shown in Table 2 . The results indicate that the $R_{\mathrm{s}}$ of the device with hexadecamer 7 is much lower than that with $7 \mathrm{a}$, this reduction correlating well with the enhancement in $J_{S C}{ }^{27,28}$ The improved performance of the device with 7 are related to the higher $\mathrm{FF}$ and $J_{\mathrm{SC}}$ and can be attributed to a better stacking in the thin film caused by intermolecular interactions by the side chains, in analogy to what has been observed for P3HT based solar cells. In particular, the large effect on $J_{\mathrm{sc}}$ values can arise from both the enhanced absorption and improved charge transport properties of planar stacked hexadecamer molecules in the crystallites. Considering the absorption spectrum of the $7 \mathrm{a}$ oligomer, it has a peak in the visible with a much lower intensity than that of 7 at an equivalent thickness of the two films; the lower absorption causes a substantial reduction in the $J_{\text {sc }}$ and in the overall performances of the device made by $7 \mathbf{a}$.

The presence of a suitable morphology in the device with compound 7 has been investigated observing the active layer by means of AFM. The AFM topography and phase images of the blend thin film with 7 are reported in Figure 6. Despite a quite high roughness (about $25 \mathrm{~nm}$ ), the 7:PCBM blend reveals the presence of a good phase separation between donor and acceptor molecules. We expect that further improvement of the device performance will be achieved by careful optimization of the processing conditions and thus control of the morphology in the active layer (see, for example, ref 29).

Attempts to obtain a solar cell with polymer 9a were unsuccessful, whereas polymer 9 showed very poor performance. Once again, it is probably the interplay of different factors that could be at the origin of these results, such as mismatches in frontier orbitals energies, low charge mobilities, or unsuitable morphology of the blends with PCBM. However, more accurate studies on the two polymers are required before a sound hypothesis can be made.

In summary, we have shown that the insertion of a sulfur atom between the thiophene ring and the alkyl side chain and the choice of the head-to-head regiochemistry-synthetically much more accessible than the head-to-tail regiochemistry for building oligomers-allows the easy preparation of a hexadecamer whose properties are similar to those of poly(3hexylthiophene), P3HT. This similarity encompasses optical, electrochemical, conductive, and even nanoscale morphology properties. We have demonstrated that the hexadecamer is a good molecular electron donor in solution-processed bulk herojunction solar cells. Initial results showed a reproducible $1.49 \%$ power conversion efficiency without any attempt to optimize processing conditions and device fabrication. We fully expect that the optimization of both material processing and device characteristics as well as the addition of appropriate additives will further improve the power conversion efficiency at the least at the maximum efficiency displayed by P3HT. Finally, these results demonstrate the viability of our approach in the engineering of novel thiophene-based molecular materials. From a synthetic point of view the approach reported here provides a simple and versatile method to obtain large size oligomers in high yield, taking advantage of ultrasound and microwave assistance.

\section{ASSOCIATED CONTENT}

\section{S Supporting Information}

All details for the preparation of the hexadecamers; ${ }^{1} \mathrm{H}$ and ${ }^{13} \mathrm{C}$ NMR spectra; details of devices. This material is available free of charge via the Internet at http://pubs.acs.org.

\section{AUTHOR INFORMATION}

\section{Corresponding Author}

*E-mail: giovanna.barbarella@isof.cnr.it.

\section{Notes}

The authors declare no competing financial interest.

\section{ACKNOWLEDGMENTS}

This research was partially supported by projects ItalNanoNet FIRB RBPR05JH2P, EFOR (L. 191/2009 art. 2 comma 44), and EU project ESCORT (n. 261920).

\section{REFERENCES}

(1) Sun, Y.; Welch, G. C.; Leong, W. L.; Takacs, C. J.; Bazan, G. C.; Heeger, A. J. Nat. Mater. 2012, 11, 44. 
(2) Brabec, C. J.; Gowrisanker, S.; Halls, J. J. M.; Laird, D.; Jia, S.; Williams, S. P. Adv. Mater. 2010, 22, 3839.

(3) Beaujuge, P. M.; Fréchet, J. M. J. J. Am. Chem. Soc. 2011, 133, 20009.

(4) Boudreault, P. L. T.; Najari, A.; Leclerc, M. Chem. Mater. 2011, 23, 456.

(5) Facchetti, A. Chem. Mater. 2011, 23, 733.

(6) Zhang, F.; Wu, D.; Xua, Y.; Feng, X. J. Mater. Chem. 2011, 21, 17590.

(7) Liang, Y.; Yu, L. Acc. Chem. Res. 2010, 43, 1227.

(8) Heeger, A. J. Chem. Soc. Rev. 2010, 39, 2354.

(9) Lin, Y.; Li, Y. F.; Zhan, X. W. Chem. Soc. Rev. 2012, 41, 4245.

(10) (a) Fitzner, R.; Mena-Osteritz, E.; Mishra, A.; Schulz, G.; Reinold, E.; Weil, M.; Körner, C.; Ziehlke, H.; Elschner, C.; Leo, K.; Riede, M.; Pfeiffer, M.; Uhrich, C.; Baüerle, P. J. Am. Chem. Soc. 2012, 134, 11064. (b) Schrader, M.; Fitzner, R.; Hein, M.; Elschner, C.; Baumeier, B.; Leo, K.; Riede, M.; Baüerle, P.; Andrienko, D. J. Am. Chem. Soc. 2012, 134, 6052. (c) Azumi, R.; Mena-Osteritz, E.; Boese, R.; Benet-Buchholz, J.; Baüerle, P. J. Mater. Chem. 2006, 16, 728.

(11) (a) Di Maria, F.; Olivelli, P.; Gazzano, M.; Zanelli, A.; Biasiucci, M.; Gigli, G.; Gentili, D.; D’ Angelo, P.; Cavallini, M.; Barbarella, G. J. Am. Chem. Soc. 2011, 133, 8654. (b) Barbarella, G.; Melucci, M.; Sotgiu, G. Adv. Mater. 2005, 17, 1581. (c) Barbarella, G.; Bongini, A.; Zambianchi, M. Macromolecules 1994, 27, 3039.

(12) (a) Jestin, I.; Frère, P.; Mercier, N.; Levillain, E.; Stievenard, D.; Roncali, J. J. Am. Chem. Soc. 1998, 120, 8150. (b) Tran, T. K.; Bricaud, Q.; Oçafrain, M.; Blanchard, P.; Roncali, J.; Lenfant, S.; Godey, S.; Vuillaume, D.; Rondeau, D. Chem.-Eur. J. 2011, 17, 5628.

(13) Bidan, G.; De Nicola, A.; Enée, V.; Guillerez, S. Chem. Mater. 1998, 10, 1052.

(14) Tanaka, S.; Tamba, S.; Tanaka, D.; Sugie, A.; Mori, A. J. Am. Chem. Soc. 2011, 133, 16734.

(15) Ng, S. C.; Mao, P. Macromolecules 1999, 32, 5313.

(16) Heinze, J.; Frontana-Uribe, B. A.; Ludwigs, S. Chem. Rev. 2010, 110, 4724.

(17) Johansson, T.; Mammo, W.; Svensson, M.; Andersson, M. R.; Inganas, O. J. Mater. Chem. 2003, 13, 1316.

(18) Hamaguchi, M.; Nambu, H.; Yoshino, K. Jpn. J. Appl. Phys. 1997, 38, L124.

(19) Arbizzani, C.; Mastragostino, M.; Zanelli, A. Sol. Energy Mater. Sol. Cells 1995, 39, 213.

(20) Kalb, W. L.; Mathis, T.; Haas, S.; Stassen, A. F.; Batlogg, B. Appl. Phys. Lett. 2007, 90, 092104.

(21) Chu, T. Y.; Lu, J.; Beaupré, S.; Zhang, Y.; Pouliot, J. R.; Wakim, S.; Zhou, J.; Leclerc, M.; Li, Z.; Ding, J.; Tao, Y. J. Am. Chem. Soc. 2011, 133, 4250.

(22) Hoppe, H.; Glatzel, T.; Niggemann, M.; Hinsh, A.; Lux-Steiner, M.Ch.; Sariciftci, N. S. Nano Lett. 2005, 5, 559.

(23) Grancini, G.; Biasiucci, M.; Mastria, R.; Scotognella, F.; Tassone, F.; Polli, D.; Gigli, G.; Lanzani, G. J. Phys. Chem. Lett. 2012, 3, 517.

(24) Heim, T.; Lmimouni, K.; Vuillaume, D. Nano Lett. 2004, 4, 2145.

(25) VanSlyke, S. A.; Chen, C. H.; Tang, C. W. Appl. Phys. Lett. 1996, 69, 2160.

(26) Osterbacka, R.; An, C. P.; Jiang, X. M.; Vardeny, Z. V. Science 2000, 287, 839.

(27) Kim, M. S.; Kim, B. G.; Kim, J. ACS Appl. Mater. Interfaces 2009, $1,1264$.

(28) Servaites, J. D.; Yenageh, S.; Marks, T. J.; Ratner, M. A. Adv. Funct. Mater. 2010, 20, 97.

(29) Seo, J. H.; Nam, S. Y.; Lee, K. S.; Kim, T. D.; Cho, S. Org. Electron. 2012, 13, 570. 